\title{
Modelling in forestry sciences, high technologies and decision-support systems in forestry and wood-processing
}

Dear readers,

We live in an extremely dynamic period characterised by enormous progress in technologies and information in all sectors, including forestry and wood-processing industry. The level of application of information and communication technologies, expert systems, and decision-making systems to the production sphere as well as to the organisational structures of enterprises is in fact considered an important criterion of the company's maturity.

Research in the forestry sector is currently aiming to support the transition to a more sustainable model that will make forests more resilient to the impacts of climate change and that will ensure more efficient use and increased valorisation of resources.

Industry 4.0 (Cyber-physical systems, Internet of Things and Services, Big data, Cloud computing, Artificial intelligence) opens up new pathways to enhance technological and economic development. However, Industry 4.0 concepts need increased and targeted efforts to be adapted in the forest research domain and the forestbased sector, to fully exploit these new opportunities for sustainable forest management and industrial growth.

The main objective of the issue is to provide insights to the state of research related to ICT technologies implementation in the Central European macro-region. It addresses main trends and priorities in three areas: 1) Forest modelling and visualisation; 2) Lidar and satellite remote sensing applications, and 3) Expert and decision support systems in forestry.

The article Forest modelling and visualisation - state of the art and perspectives represents a perfect introduction to the first section. It brings an evaluation of the current state of technical possibilities, perspectives of modelling and visualisation of forest ecosystems. In the example of 34 forest models, it demonstrates an integrated approach for model categorisation using a classification scheme. Forest visualisation is described from the point of different visualisation methods and used technologies. Data inputs that are necessary for the models and need to be derived using specialised tools, such as various forms of data generators, are presented, too. Perspectives and challenges for further development of forest models and visualisation technologies were specified as well.

The representative article Fir (Abies spp.) stand biomass additive model for Eurasia sensitive to winter temperature and annual precipitation is an excellent example of data integration from studies of 272 fir stands in Eurasia and an advanced method of biomass modelling by a three-step proportional weighting additive model disaggregating tree biomass into individual tree compartments. The results indicate that fir seems to be a perspective taxon from the point of its productive properties in the ongoing process of climate change.

Historical radial increment data from the close-to-nature experimental forest management unit in Central Slovakia was used for retrospective modelling of changes inforest dynamics using an advanced density-dependent matrix transition model. An integrated tool for nonlinear financial optimisation searched for an optimal management equilibrium represented by optimal basal area, tree species composition, diameter distribution and target harvest diameter. The main lesson learnt from the past is not to trust simple extrapolation of current trends, such as the observed continual decline of spruce related to climate change, too much, but to be aware of temporal and possibly reversible processes, such as the observed extensive fir recovery after reduction of air pollution. Tree species diversity appears to be the best option for the uncertain future.

The second section comprises four papers about close-range photogrammetry, airborne lidar scanning and MODIS satellite data. Contributions point at the widespread use of remote sensing data to detect tree, stand, ecological and geographical characteristics from a tree level to large-scale applications.

The study Predicting forest stand variables from airborne LiDAR data using a tree detection method in Central European forests is touching the future. It is a nice example of a more accurate estimation of stand characteristics (mean height, mean diameter and total stock) using a strictly scientific method based on new software solutions. Specifically, the multisource-based method, implemented in reFLex software, uses all the information contained in the original point cloud and a priori information. 
Each new technology requires verification of its accuracy, and each increase in accuracy increases the value of new technology. In this sense, the highly valuable study is The vertical accuracy of digital terrain models derived from the close-range photogrammetry point cloud using different methods of interpolation and resolutions. The research results proved further possibilities and improvements in generating digital terrain models (DTM) using techniques of structure-from-motion and multi-view stereo.

The essence of ecosystem research is their long-term and continuous monitoring. The paper Suitability of MODIS-based NDVI index for forest monitoring and its seasonal applications in Central Europe demonstrates the multipurpose application of the normalised difference vegetation index (NDVI) derived from MODIS products for forest damage monitoring, forest phenology and increment assessment in relation to climate indices across the macro-region of the Western Carpathians and Pannonian basin.

Wood assessment optimisation should be the top priority of the forestry subjects that are fundamentally dependent on the income from its sale. The paper Possibilities of image analysis for quality wood sorting compared the classical methodology used in forestry practice with the application of ImageJ software to determine false heartwood and rot. The ImageJ software application led to an improved assessment (transfer to a higher quality class) in $56 \%$ of the logs. The analysis therefore confirmed that in the case of a considerable irregularity in a qualitative character (when the surface area of the character significantly differs from the circumscribed circular surface), the standard STN EN 1309-3 methodology systematically overvalues the surface area of this character.

The third section devoted to the research in the area of expert and decision support systems covers two papers, which confirm a tendency from description to forecasting and multi-disciplinarity of the solution related to forest management planning and wood marketing.

The first one is focused on Marketing support of decision-making at the forest enterprise: A case study on roundwood assortments portfolio. The paper compared the most used marketing decision-making models: SWOT, Growth-share matrix BCG and Mckinsey GE matrix with the support of ABC analysis. The outcomes of models contribute to each other and do not contradict. The methods of marketing decision-making models can be applied in managing forest enterprises and they are a great contribution. However, the experience and knowledge of a marketer or a manager are important for the correct interpretation of these models.

Ecosystem services play an important role in the daily life and well-being of millions of people worldwide. The paper Multi-objective land allocation for zoning of ecosystem services in mountain forests describes and proposes an innovative four-phase workflow of the evaluation of forest ecosystem services and of the zoning of priority areas integrating the knowledge system and analytical functionalities of GIS. Evaluated are erosion control, avalanche control, wood production and cultural services. Techniques of multi-objective land allocation were applied to allocate complementary and conflicting objectives. The non-financial system utilising fuzzy logic for evaluation purposes proved to be appropriate to compare the priorities of individual ecosystem services. A comparison of results with the existing (control) map of ecosystem services has proved that the proposed system is a potent means for multi-objective forest planning.

We thank all authors for their contributions and professional approach to presenting their research areas and the latest trends. We are aware of the fact that the issue of the journal is far from covering the whole range of research and innovation activities related to the implementation of modern technologies in forestry, such as computerisation of analogue reports and records, modernisation of web applications into responsive widely available applications, mobility of field data collection applications etc. In spite of this we believe that the presented issue of the journal will provide the professional public with useful and interesting insights into the current development and the state of informatics and modern technologies in forestry and forest-based industry and thus contribute to further development of our sector.

Dr. Ing. Tomáš Bucha

Member of Editorial Board

Director of National Forest Centre - Forest Research Institute Zvolen 